\title{
An unusual phenotype of acute aortic syndrome: A case report.
}

\author{
Mariane Higa Shinzato', Renato A Hortegal ${ }^{1 *}$, A. Tito Paladino F $^{1}$,Pablo Santos Graffitti ${ }^{1}$, Rafael Nunes \\ de Oliveira ${ }^{1}$, Rodrigo Augusto de Miranda Bertin ${ }^{1}$, Diandro Marinho Mota', Hugo Ribeiro Ramadan', \\ Ibraim Masciarelli ${ }^{2}$, Louis Nakayama Ohe ${ }^{1}$
}

'Department of Cardiovascular Emergency, Dante Pazzanese Institute of Cardiology, São Paulo, Brazil

${ }^{2}$ Department of Cardiovascular Imaging, Dante Pazzanese Institute of Cardiology, São Paulo, Brazil

\begin{abstract}
Acute Aortic Syndromes are a major cause of acute chest pain. We report a case of a woman which presented mixed features from acute aortic syndromes phenotypes in angiotomography.

Keywords: Acute aortic syndrome, Angiotomography, Myocardial infarction.
\end{abstract}

Accepted on July 21, 2020

\section{Case Presentation}

An 80-year-old woman presents to the emergency room complaining about a high-intensity chest pain. She was admitted 12 hours after the pain onset, maintaining the same characteristics, excruciating and continuous. The pain arose after physical activity (dance), radiating to the shoulders. The patient denied sweating and dyspnea. She referred that the pain worsened with inspiration and had no improvement factor. On physical examination, the patient was admitted normocardial and normotensive, with no signs that attracted attention except for the facies of pain. Peripheral pulses present and symmetrical. A 12-lead ECG and blood test for troponin are negative for acute myocardial infarction.

\section{Past Medical History}

The patient's past medical history included hypertension and rheumatoid arthritis.

\section{Differential Diagnosis}

Differential diagnoses of acute chest pain are broad and include acute coronary syndrome, pulmonary embolism, acute aortic syndrome, spontaneous pneumotorax esophageal, rupture as the main malignant causes.

\section{Investigations}

The X-ray showed enlarged mediastinum on admission. The transthoracic echocardiogram performed showed a significant increase in the ascending aorta $(52 \mathrm{~mm})$. Aortic root measured $35 \mathrm{~mm}$, aortic arch $28 \mathrm{~mm}$ and proximal abdominal aorta 20 $\mathrm{mm}$. An echogenic image was observed in the ascending aorta with $9 \mathrm{~mm}$ and in less curvature of the aortic arch, corresponding to an intramural hematoma.

For diagnostic confirmation, aortic angiotomography was performed (Figure 1). The intramural hematoma extended from the root to the abdominal segment of the aorta ( $44 \mathrm{~mm}$ below the thoracoabdominal junction, with a larger diameter of $56 \times$ $61 \mathrm{~mm}$. There was an increase in the thickness of the aortic wall, which means it measured $12 \mathrm{~mm}$ in the largest diameter.
An ulcer was seen on the aortic wall $20 \mathrm{~mm}$ above the sinotubular junction (Figure 1, image $\mathrm{A}$ and $\mathrm{B}$, on the white arrow). Presence of peri-aortic collection along the ascending aorta was visualized (Figure 1, image $\mathrm{A}$ and $\mathrm{B}$, on the blue arrow). Mild pericardial effusion and bilateral pleural effusion, with signs of compression atelectasis were also observed.
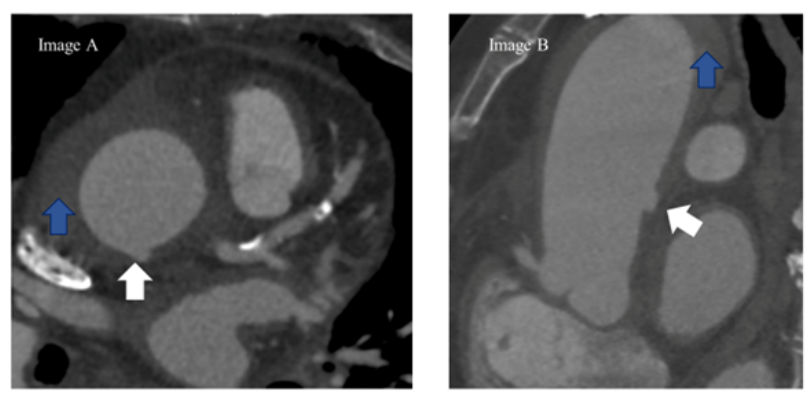

Figure 1. First angiotomography performed. White arrow (image A), presence of penetrating aortic ulcer.Blue arrow (image A), presence of aortic intramural haematoma. White arrow (image B), presence of penetrating aortic ulcer.Blue arrow (image B), presence of aortic intramural haematoma.

In the brachiocephalic trunk, intramural hematoma was seen in the proximal third, measured $21 \times 19 \mathrm{~mm}$ at the largest diameter (extension of the hematoma seen in the aorta- Figure 2). The hematoma involves the proximal segment of right coronary artery.

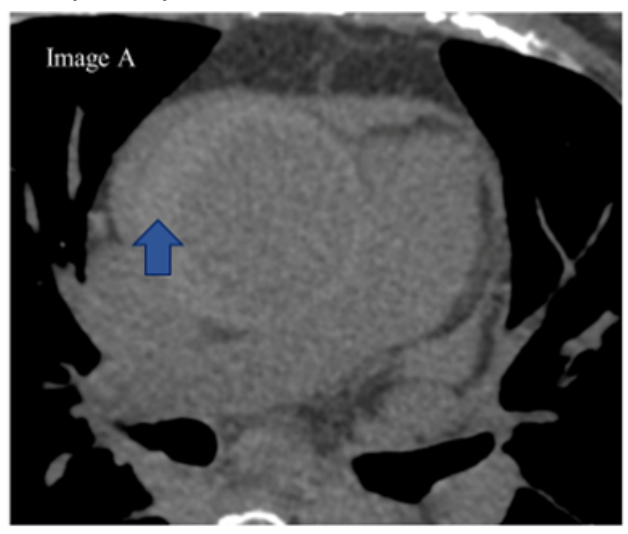

Figure 2. Presence of aortic intramural haematoma.Blue arrow (Image A), presence of aortic intramural haematoma on the first tomography. 


\section{Management (Medical/Interventions)}

At first, due to the high surgical risk (Euroscore 14,96\% and STS $5,723 \%$ ), the heart team cardiac surgery team opted for a conservative approach. However, 13 days later, control angiotomography was requested, which showed an increase in ulcer longitudinal extension to 4,7 cm (Figures 3 and 4).
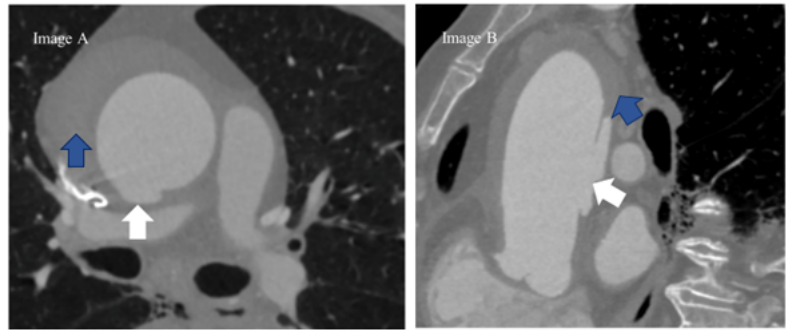

Figure 3. Control angiotomography. White arrow (image A), presence of penetrating aortic ulcer.Blue arrow (image A), presence of aortic intramural haematoma. White arrow (image $B)$, presence of penetrating aortic ulcer showing an important increase to $4,7 \mathrm{~cm}$ in longitudinal extension. Blue arrow (image B), presence of aortic intramural haematoma.

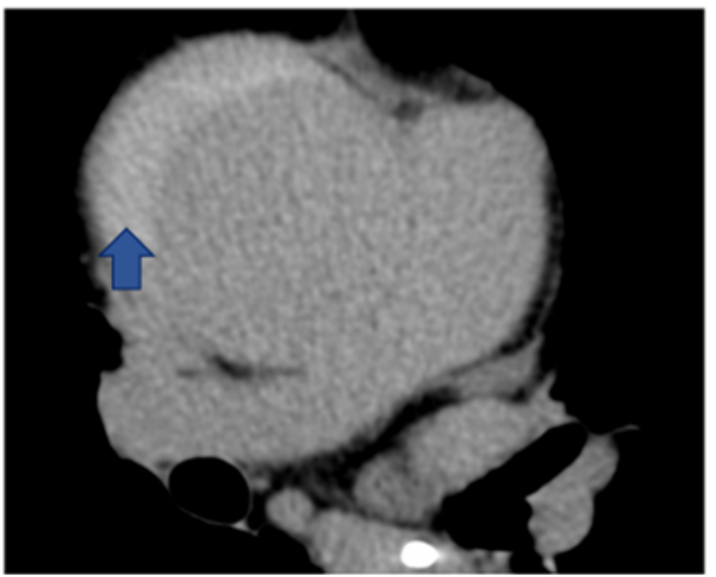

Figure 4. Presence of aortic intramural haematoma.Blue arrow, presence of aortic intramural haematoma on the control angiotomography.

After angiotomography control, the heart team opted for a surgical approach. On the fourteenth day of hospitalization, surgery was performed with placement of a supra- coronary tube, without coronary tube, without intervening the aortic valve.

\section{Discussion}

Acute Aortic Syndromes (AAS) are a group of medical emergencies with similar clinical presentation, which are characterized by the injury to the aortic wall with different profiles of structural breakdown.

Since the initial clinical picture of AAS might not be too different from other differential diagnosis such as the acute coronary syndromes and the pulmonary embolism as well as other malignant causes of chest pain, the imaging methods are essential for the diagnosis.
The characteristics of the chest pain (sudden onset and excruciating) and/or the presence of increased diameter of the thoracic aorta on chest radiography (seen in $90 \%$ of the cases) are the main red flags that allow us to suspect AAS, order the complementary tests and avoid DAPT.

The angiotomography, magnetic resonance and transesophageal echocardiography have similar accuracy, and the imaging modality choice depends on the patient's clinical condition, the physician's preference and the availability of the method. Currently, angiotomography has been the imaging method of choice in diagnosis and follow-up, despite the need of intravenous contrast [1].

Our patient presented features from different AAS phenotypes: a large intramural hematoma, an extensive longitudinal propagation that remains dissection, but without evidence of intimal tear. Also, two large PAU were found: one in proximal aorta and a second one in descendent aorta.

We were not able to determine precisely which one of these findings was the main trigger, even though the coexistence of those different types of lesions to the aortic wall suggests an advanced degree of vessel structure disarrangement [2].

Since the aortic architecture is given by the balance between the shear forces and aortic strength, one hypothesis is that the extensive intramural hematoma lead to a reduction in aortic strength and then to a further weakening the aortic wall which favored this atypical progression increase of PAU observed in the control CT 13 days after the admission.

These conceptual and pathophysiological issues have some practical implications since the type A aortic dissection requires prompt surgical intervention while for the other modalities (penetrating ulcer and intramural hematoma) the invasive management still remains less well established.

After the initial clinical support aiming the reduction of blood pressure to values $100-120 \mathrm{mmHg}$ and heart rate to $<60 \mathrm{bpm}^{2}$, ESC (European Society of Cardiology) 2014, on cases of type indicated and urgent surgery (24 hours after diagnosis) is required in most of Type A IMHs complicated with pericardial effusion, periaortichaematoma, or large aneurysms.

In elderly patients or those with significant comorbidities, initial medical treatment with a 'wait-and-watch strategy' (optimal medical therapy with blood pressure and pain control and repetitive imaging) may be a reasonable option, particularly in the absence of aortic dilation $(<50 \mathrm{~mm})$ and IMH thickness, $<11 \mathrm{~mm}^{3}$.

The treatment recommendations for PAUS are poorly mentioned. Since these patients are often poor candidates for conventional surgery due to advanced age and related comorbidities - and the aortic lesions, due to their segmental nature, represent an ideal anatomical target for stentingTEVAR is increasingly being used for this indication, with encouraging results [3-11]. 


\section{Follow-up}

In the immediate postoperative period, the patient developed atrial fibrillation without hemodynamic instability. Empirical antibiotic therapy was started on the fourth postoperative day due to fever. She underwent 6 days of antibiotic therapy with significant clinical improvement. The patient was discharged on the 12th postoperative day with normal infectious parameters. Currently, she follows an outpatient routine.

\section{Conclusion}

Intramural aortic hematoma and penetrating aortic ulcer are unusual conditions. Currently, cardiac angiotomography plays a primary role in primary diagnosis, as well as evolutionary control and therapeutic planning.

In this case, we show the evolution of an intramural hematoma and penetrating aortic ulcer. It must be considered that the hematoma itself can be considered as a factor of fragility in the aortic wall, which may have contributed to an increase in the size of the ulcer (which quadrupled in the longitudinal direction).

\section{Learning Objective}

- To present and discuss a case with feature overlapping of different modalities of acute aortic syndrome.

- Review the tomographic findings of intramural hematoma and penetrating aortic ulcer.

- Elucidate possible evolution of an intramural hematoma and penetrating aortic ulcer.

\section{References}

1. Hiratzka LF, Bakris GL, Beckman JA, et al. Guidelines for the diagnosis and management of patients with thoracic aortic disease. Circulation. 2010; 121:266-369.

2. Erbel R, Aboyans V, Boileau C, et al. 2014 ESC Guidelines on the diagnosis and treatment of aortic diseases: Document covering acute and chronic aortic diseases of the thoracic and abdominal aorta of the adult. The Task Force for the Diagnosis and Treatment of Aortic Diseases of the European Society of Cardiology (ESC). Eur Heart J. 2014;35(41):2873-926.

3. Mussa FF, Horton JD, Moridzadeh R, et al. Acute Aortic Dissection and Intramural Hematoma: A Systematic Review. JAMA. 2016;316(7):754-63.

4. Navarro-Triviño FJ, Cuenca-Manteca J, Ruiz-Villaverde R. Allergic contact dermatitis with systemic symptoms caused by VenaSeal. Contact Dermatitis. 2020;82(3):185 - 187.

5. Tang YT, Rathnaweera HP, Kam JW, et al. Endovenous cyanoacrylate glue to treat varicose veins and chronic venous insufficiency-Experience gained from our first $100+$ truncal venous ablations in a multi-ethnic Asian population using the Medtronic VenaSeal TM Closure System. Phlebology. 2019;34(8):543-551.
6. Jones AD, Boyle EM, Woltjer R, et al. Persistent type IV hypersensitivity after cyanoacrylate closure of the great saphenous vein. J VascSurg Cases Innov Tech. 2019;5(3): $372-374$.

7. Gibson K, MinjarezR,Rinehardt E, et al. Frequency and Severity of Hypersensitivity Reactions in Patients After VenaSeal ${ }^{\text {TM }}$ Cyanoacrylate Treatment of Superficial Venous Insufficiency. Phlebology. 2020;35(5):337-344.

8. Parsi K, Roberts S, Kang M, et al. Cyanoacrylate Closure for Peripheral Veins: Consensus Document of the Australasian College of Phlebology. Phlebology . 2020;35(3):153-175.

9. Langridge BJ, Onida S, Weir J, et al. Cyanoacrylate Glue Embolisation for Varicose Veins - A Novel Complication. Phlebology. 2020;268355520901662.

10. Quirce S, Baeza ML, Tornero P, et al. Occupational Asthma Caused by Exposure to Cyanoacrylate. Allergy. 2001;56(5): $446-449$.

11. Shaĭdakov EV, Mel'tsova AZ, Porembskaia OI, et al. Experience with using cyanoacrylate glue in endovascular treatment of varicose veins. Angiology and vascular surgery. 2017;23(4):62-67.

\section{*Correspondence to}

Renato A Hortegal

Department of Cardiovascular Emergency

Dante Pazzanese Institute of Cardiology

São Paulo

Brazil

E-mail: renato.hortegal@dantepazzanese.org.br 